\title{
Wavelet Variance, Covariance and Correlation Analysis of BSE and NSE Indexes Financial Time Series
}

\author{
Anuj Kumar ${ }^{1 *}$, Sangeeta Pant ${ }^{1}$, Lokesh Kumar Joshi ${ }^{2}$ \\ ${ }^{1}$ Department of Mathematics, University of Petroleum \& Energy Studies, Dehradun-248007, India \\ ${ }^{2}$ Department of Applied Mathematics, Faculty of Engineering and Technology \\ Gurukul Kangri Vishwavidyalaya, Haridwar-249404, India \\ ${ }^{*}$ Corresponding author: anuj4march@gmail.com
}

(Received February 26, 2016; Accepted March 8, 2016)

\begin{abstract}
The mostly used measure to analyze the stock market behavior is wavelet correlation analysis. Cross-country correlations have been largely used to obtain a static estimate of the comovements of actual returns across country. In this paper wavelet based variance, covariance and correlation analysis of BSE and NSE indexes financial time series have been done using index data from April 1990 to March 2006.
\end{abstract}

Keywords-stock markets, MODWT, financial time series, BSE \& NSE indexes, wavelet variance and covariance.

\section{Introduction}

A time series is a sequence of data points, measured typically at successive times, spaced at uniform time intervals. Time series analysis comprises methods that attempt to understand such time series, often either to understand the underlying theory of data points or to make forecasts (predictions). Examples of time series are the gross national product, steel production, income per capita, etc. Wavelets are a relatively new way of analyzing time series. Wavelet analysis is in some cases complementary to existing analysis techniques (e.g. correlation and spectral analysis) and in cases capable of solving problems for which little progress has been made prior to the introduction of wavelets (Percival and Walden, 2000).

Traditional time series analysis techniques can be represented as autoregressive integrated moving average models (Bowerman and Connell, 1987; Box and Jenkins, 1976). The traditional models can provide good results when the dynamic system under investigation is linear or nonlinear. However, for cases in which the system dynamics are highly nonlinear, the performance of traditional models might be very poor (Cichocki and Unbehauen, 1993; Weigend and Gershenfeld, 1994). The analysis of time series has often been difficult when data do not conform to well study theoretical concepts. One of the most common statistical properties violated by time series data is stationarity. A time series is considered (weakly or second-order) stationary when it has a mean and auto covariance sequence that do not vary with time. It is not uncommon to encounters departures from stationarity in financial time series. Its effects are not limited to the mean of a financial time series, but may also enter into the variance. Other time series exhibit a persistence of correlation much longer than can be explained by short memory (ARIMA) models; they are known as long memory process. The existence of data, such as these, that defy current statistical methods motivates researchers to develop better theories and better tools with which to analyze them. Another concept which arises in the time series analysis is the notion of 'multiscale features' i. e. , an observed financial time series may contain several phenomena, each occurring in different time scales (these correspond to ranges of frequencies in the Fourier 
International Journal of Mathematical, Engineering and Management Sciences

Vol. 1, No. 1, 26-33, 2016

https://dx.doi.org/10.33889/IJMEMS.2016.1.1-003

domain). Wavelet techniques possess a natural ability to decompose financial time series into several sub-series which may be associated with particular time scales. Hence, interpretation of features in complex financial time series may be alleviated by first applying a wavelet transform and subsequently interpreting each individual sub-series (Kumar et al., 2010, 2011).

\section{Methodology}

Variability and association structure of certain stochastic processes can be represented with the help of wavelet methods on a scale-by-scale basis. For a given stationary process $\{X\}$ with variance $\sigma_{X}^{2}$, the wavelet variance $\sigma_{X, j}^{2}$ at scale $j$ have the relationship (Saiti et al., 2014):

$\sum_{j=1}^{\infty} \sigma_{X, j}^{2}=\sigma_{X}^{2}$

Thus, as $\sigma_{X, j}^{2}$ represents the contribution of the changes at scale $j$ to the overall variability of the process.

With the help of the above relationship the variance of a time series can be decomposed into components that are associated to different time scales. Spectral density decomposes the variance of the original series with respect to frequency $f$ in the similar manner the wavelet variance decomposes the variance of a stationary process with respect to the scale at $j^{\text {th }}$ level i.e.

$\sum_{j=1}^{\infty} \sigma_{X, j}^{2}=\sigma_{X}^{2}=\int_{-1 / 2}^{1 / 2} S_{X}(f) d f$

where $S($.$) denotes the spectral density function.$

By definition the time independent wavelet variance at scale $j, \sigma_{X, j}^{2}$ is given by the variance of $j$ level wavelet coefficients $\sigma_{X, j}^{2}=\operatorname{var}\left\{\tilde{W}_{j, t}\right\}$.

A time-independent wavelet variance may be defined not only for stationary processes but also for non-stationary processes with stationary $d^{\text {th }}$ order differences with local stationarity (Gallegati, 2008). As the wavelet filter $\left\{h_{l}\right\}$ represents the difference between two generalized averages and is related to a difference operator, wavelet variance is time-independent in case of non-stationary processes with stationary $d^{\text {th }}$ order differences, provided that the length $L$ of the wavelet filter is large enough. $L \geq d$ is a sufficient condition to make the wavelet coefficients $\tilde{W}_{j, t}$ of a stochastic process stationary whose $d^{\text {th }}$ order backward difference is stationary.

As MODWT employs circular convolution, the coefficients generated by both beginning and ending data could be spurious. Thus, if the length of the filter is $L$, there are $\left(2^{j}-1\right)(L-1)$ coefficients affected for $2^{j-1}$-scale wavelet and scaling coefficients (Schleer-van and Gellecom, 2014). If $N-L_{j} \geq 0$, then an unbiased estimator of the wavelet variance based on the MODWT may be obtained by removing all coefficients affected by the periodic boundary conditions using $\tilde{\sigma}_{X, j}^{2}=\frac{1}{\tilde{N}_{j}} \sum_{t=L_{j}}^{N} \tilde{W}_{j, t}^{2}$, where $\tilde{N}_{j}=N-L_{j}+1$ is the number of maximal overlap coefficients at 
International Journal of Mathematical, Engineering and Management Sciences

Vol. 1, No. 1, 26-33, 2016

https://dx.doi.org/10.33889/IJMEMS.2016.1.1-003

scale $j$ and $L_{j}=\left(2^{j}-1\right)(L-1)+1$ is the length of the wavelet filter for level $j$. Thus, the $j^{\text {th }}$ scale wavelet variance is simply the variance of the non-boundary or interior wavelet coefficients at that level. Scaling of BSE index financial time series has been done and shown that it is monofractal and can be represented by a fractional Brownian motion (Razadan, 2002).

The MODWT-based estimator has been shown to be superior to the DWT-based estimator although both can decompose the sample variance of a time series on a scale-by-scale basis via its squared wavelet coefficients.

To determine the magnitude of the association between two financial time series of observations $X$ and $Y$ on a scale-by-scale basis the notion of wavelet covariance is used. The wavelet covariance at wavelet scale $j$ can be defined as the covariance between scale $j$ wavelet coefficients of $X$ and $Y$, i.e.

$\tilde{\gamma}_{X Y, j}=\operatorname{cov}\left[\tilde{W}_{j, t}^{X} \tilde{W}_{j, t}^{Y}\right]$

An unbiased estimator of the wavelet covariance using MODWT can be obtain by removing all wavelet coefficients affected by boundary conditions and given by

$\tilde{\gamma}_{X Y, j}=\left(\frac{N-1}{\tilde{N}_{j}}\right) \sum_{t=L_{j}-1}^{N-1} \tilde{W}_{j, t}^{X} \tilde{W}_{j, t}^{Y}$

The MODWT estimator of the wavelet cross-correlation coefficients for scale $j$ and $\operatorname{lag} \tau$ may be obtained by making use of the wavelet cross-covariance $\tilde{\gamma}_{\tau, X Y, j}$, and the square root of the wavelet variances $\tilde{\sigma}_{X, j}$ and $\tilde{\sigma}_{Y, j}$ by

$\tilde{\rho}_{\tau, X Y, j}=\frac{\tilde{\gamma}_{\tau, X Y, j}}{\tilde{\sigma}_{X, j} \tilde{\sigma}_{Y, j}}$

The wavelet cross-correlation coefficients $\tilde{\rho}_{\tau, X Y, j}$, just as the usual unconditional crosscorrelation coefficients are between 0 and 1 and provide the lead/lag relationships between the two processes on a scale-by-scale basis.

Starting from spectrum $S \omega_{X, j}$ of scale $j$ wavelet coefficients, it is possible to determine the asymptotic variance $V_{j}$ of the MODWT-based estimator of the wavelet variance and construct a random interval which forms a $100(1-2 p) \%$ confidence interval.

\section{Results and Conclusion}

Here, the authors have presented the variance of a process on a scale basis with the help of wavelet analysis. Plot of $\tilde{\sigma}_{X, j}^{2}$ against scale $j$ indicates which scales contribute more to the process variance. Fig. 1 shows the MODWT-based variance of the BSE Index and NSE Index plotted on a log-log scale. In this figure the straight line " $U$ " and " $L$ " represent the upper and lower bounds for the 95\% approximate confidence interval and the straight line shows the valued wavelet variance. Reflection boundary condition has been applied for the calculation of wavelet 
International Journal of Mathematical, Engineering and Management Sciences

Vol. 1, No. 1, 26-33, 2016

https://dx.doi.org/10.33889/IJMEMS.2016.1.1-003

variance. Due to this, we have sufficient number of non-boundary coefficients to approximate wavelet variance up to scale 6 .

As wavelet analysis have the ability to decompose a financial time series into its time scale components. It is also advantageous in analyzing conditions in which the degree of association between two financial time series is likely to change with the time-horizon. The lead/lag relationship between two financial time series of BSE and NSE has been analyzed on a scale-by-scale source by using wavelet cross-correlation analysis.
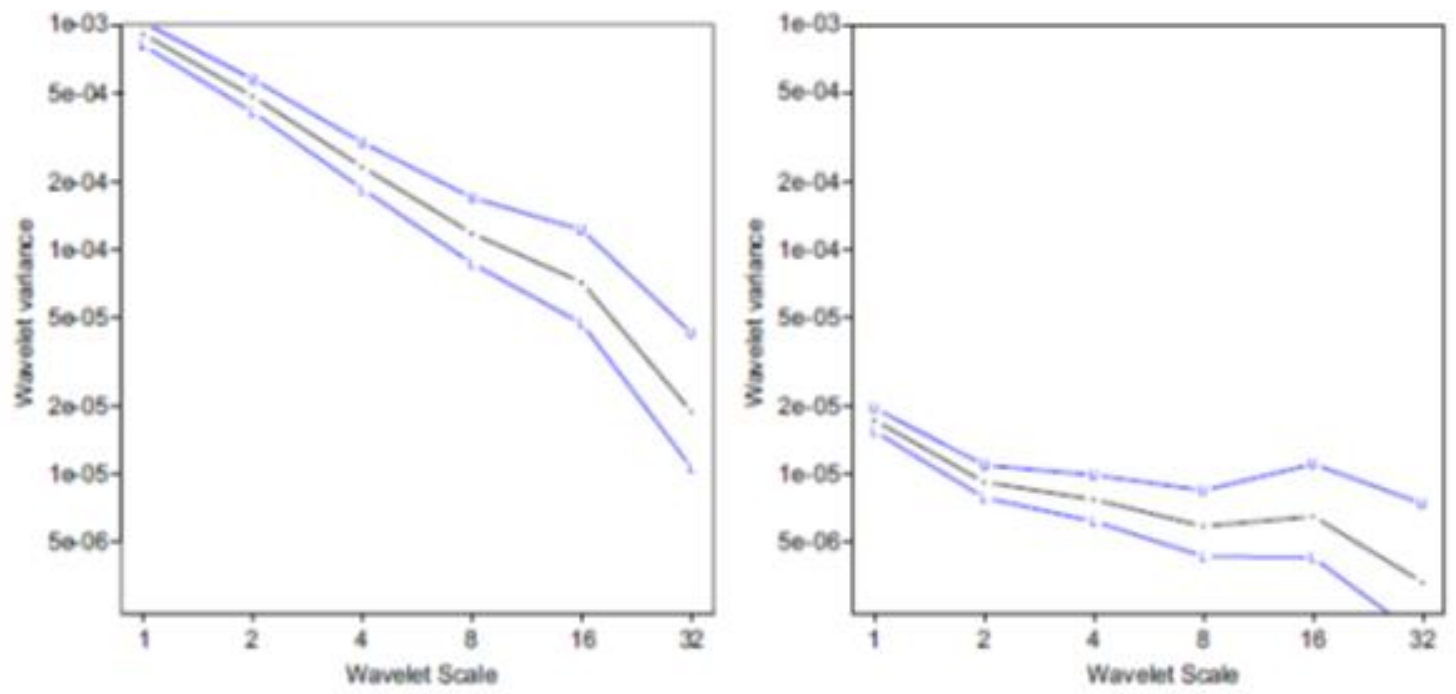

Fig. 1. Wavelet variance for the BSE index and NSE index at log-log scale

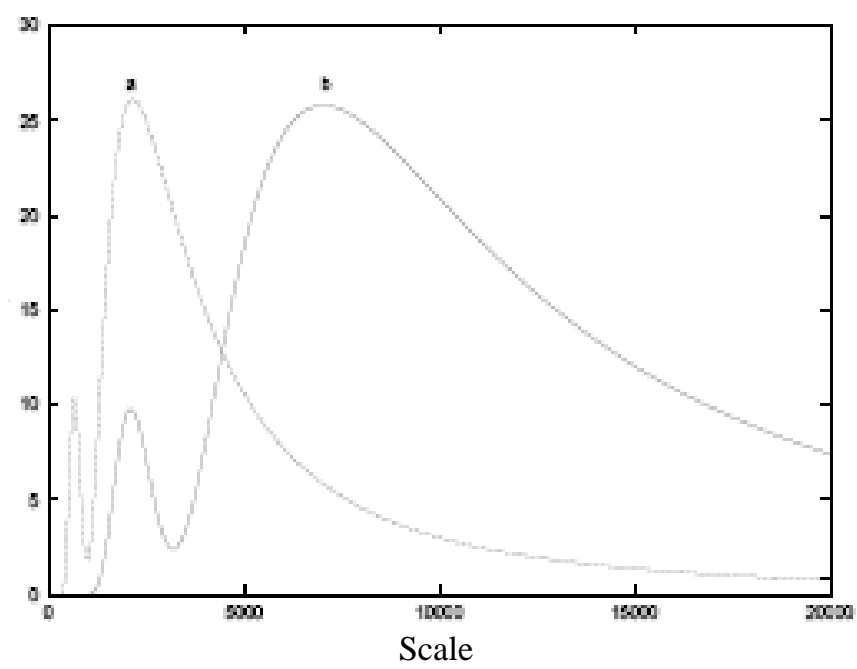

Fig. 2. Wavelet spectrum of NSE (curve a) and BSE (curve b) indices 
International Journal of Mathematical, Engineering and Management Sciences

Vol. 1, No. 1, 26-33, 2016

https://dx.doi.org/10.33889/IJMEMS.2016.1.1-003

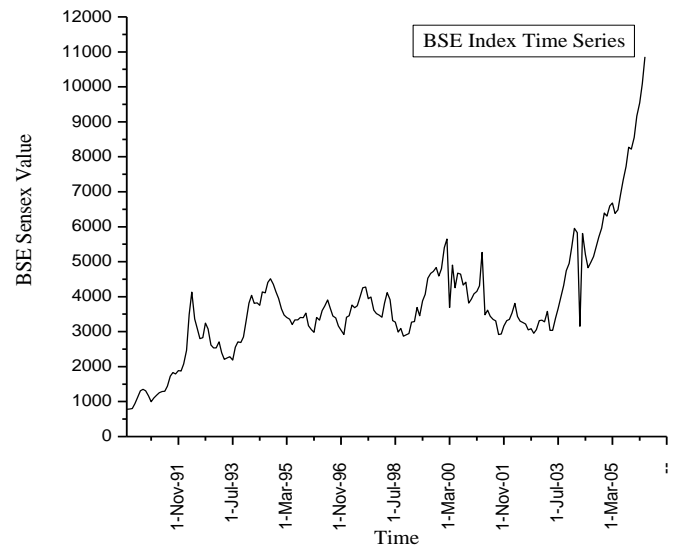

Fig. 3. Financial time series of BSE index

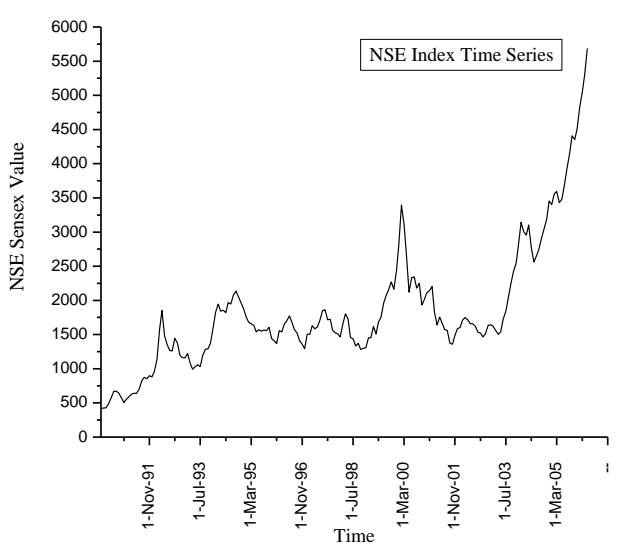

Fig. 4. Financial time series of NSE index

Fig. 2 shows the Wavelet spectrum of NSE and BSE indices and Fig. 3 \& Fig. 4 shows the financial time series of BSE and NSE indexes. Fig. 5 exhibits the MODWT-based wavelet correlations and cross-correlation coefficients with the corresponding imprecise confidence intervals. For example, scale 1 is associated to 2-4 month periods, scale 2 to $4-8$ month periods, scale 3 to $8-16$ month periods, and so on.

The magnitude of the association between the two variables at the shortest scales; i.e. scales 1 to 2 ; is generally close to zero at all leads and lags, whereas at scales 4 and 5 , such connection become stronger. There is a low magnitude of association between BSE and NSE indexes at scales 3 and 4 as the value of wavelet correlation coefficient at lag zero indicates.

On the other hand, the cross-correlation wavelet coefficients 0.5 and 0.7 at scale 4 and 5 reveal high positive foremost relationship between BSE and NSE indexes with the leading period increasing as the time scale increases. It is clear that the largest cross-correlation coefficients going on at leads 6 for wavelet scale 4, that is 16-32 month periods, and 10 for wavelet scale 5, that is $32-64$ month periods.

Table 1 and Table 2 represents the monthly average of BSE and NSE Sensex closing index

The averages are based on daily BSE Sensex closing index.

(Base: $1978-79=100)$

The averages are based on daily closing index.

(Base: 1983-84 = 100)

So, it is clear from the above discussion that correlation between BSE and NSE indices is scale dependent. 
International Journal of Mathematical, Engineering and Management Sciences

Vol. 1, No. 1, 26-33, 2016

https://dx.doi.org/10.33889/IJMEMS.2016.1.1-003
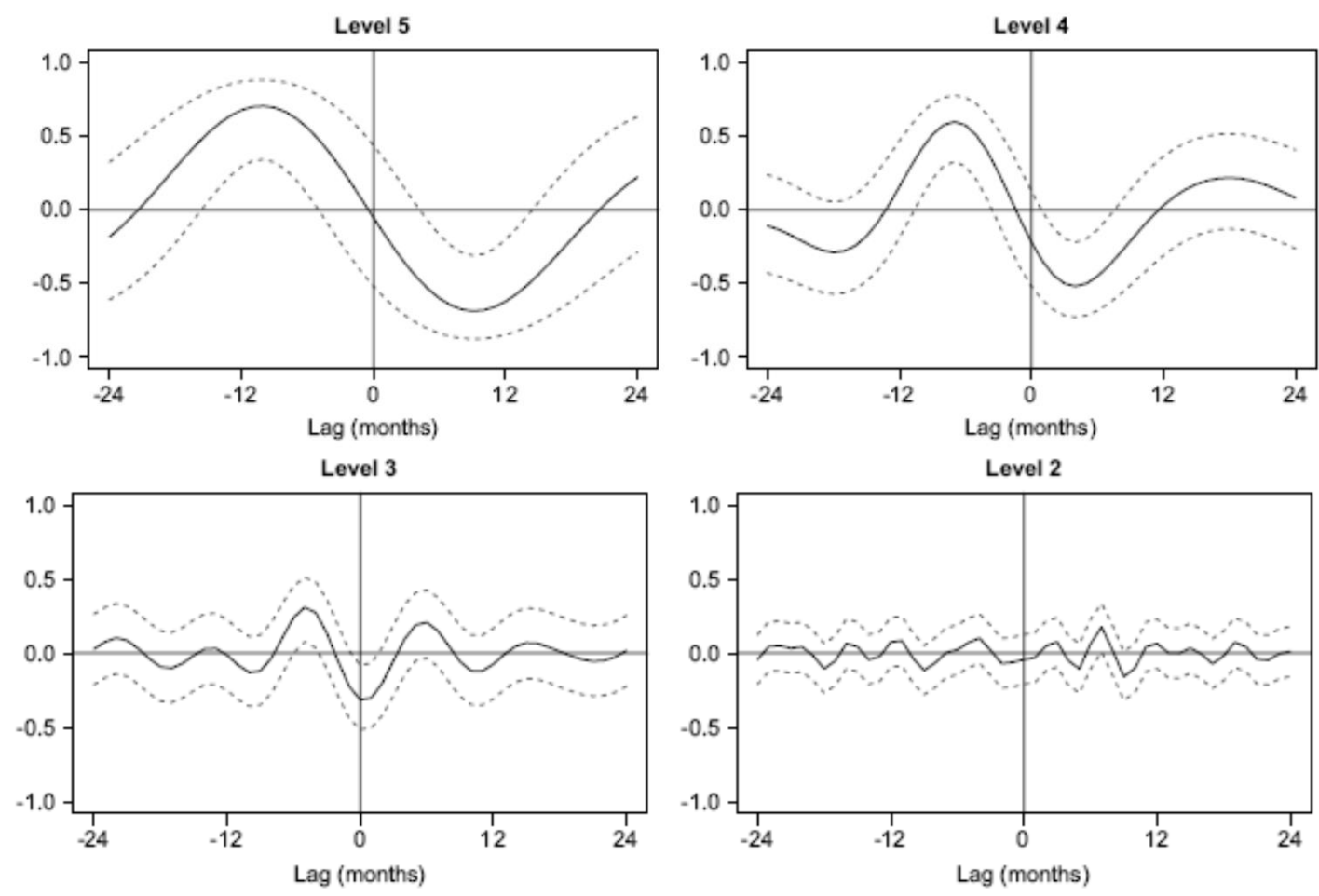

Level 1

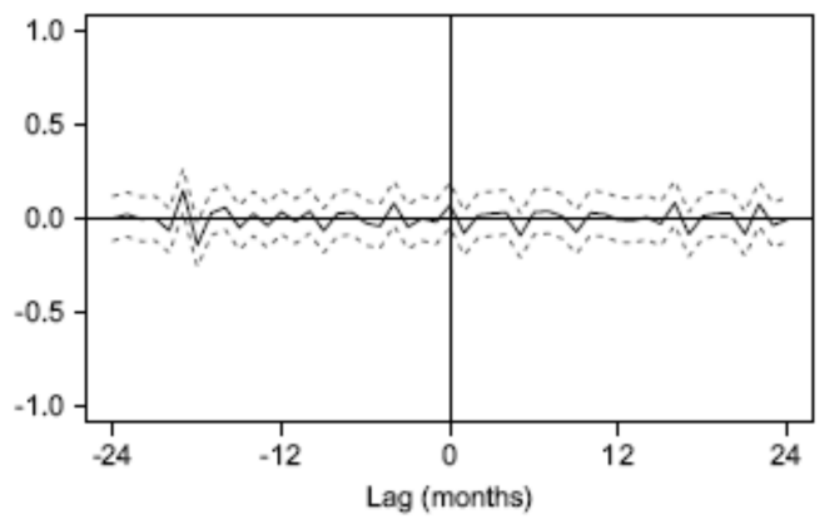

Fig. 5. Wavelet cross-correlations between BSE and NSE indexes 
International Journal of Mathematical, Engineering and Management Sciences

Vol. 1, No. 1, 26-33, 2016

https://dx.doi.org/10.33889/IJMEMS.2016.1.1-003

\begin{tabular}{|l|l|l|l|l|l|l|l|l|l|l|l|l|}
\hline Year/Month & Apr. & May & Jun. & Jul. & Aug. & Sept. & Oct. & Nov. & Dec. & Jan. & Feb. & Mar. \\
\hline 1 & 2 & 3 & 4 & 5 & 6 & 7 & 8 & 9 & 10 & 11 & 12 & 13 \\
\hline $1990-91$ & 780.18 & 785.57 & 802.45 & 938 & 1116.19 & 1307.87 & 1354.02 & 1306.09 & 1161.87 & 996.45 & 1100.78 & 1180.7 \\
\hline $1991-92$ & 1255.25 & 1291.74 & 1295.15 & 1440.72 & 1723.82 & 1833.34 & 1789.5 & 1890.09 & 1872.31 & 2073.6 & 2464.74 & 3487.19 \\
\hline $1992-93$ & 4131.01 & 3366.55 & 3088.59 & 2797.27 & 2829.96 & 3243.19 & 3075.28 & 2618.2 & 2535.64 & 2532.86 & 2708.72 & 2398.27 \\
\hline $1993-94$ & 2205.37 & 2248.01 & 2281.95 & 2190.34 & 2556.16 & 2708.39 & 2688.51 & 2850.35 & 3301.85 & 3813.74 & 4039.42 & 3811.25 \\
\hline $1994-95$ & 3824.75 & 3756.1 & 4135.67 & 4106.95 & 4407.4 & 4511.34 & 4351.16 & 4139.06 & 3949.78 & 3651.59 & 3474.92 & 3408.29 \\
\hline $1995-96$ & 3359.29 & 3206.86 & 3336.46 & 3334.86 & 3402.81 & 3396.37 & 3528.1 & 3172.02 & 3060.05 & 2979.3 & 3405.56 & 3327.33 \\
\hline $1996-97$ & 3599.66 & 3732.2 & 3906.72 & 3668.21 & 3449.17 & 3390.11 & 3159.79 & 3044.28 & 2918.68 & 3410.3 & 3453.24 & 3762.52 \\
\hline $1997-98$ & 3681.5 & 3740.95 & 4001.47 & 4256.11 & 4276.31 & 3944.79 & 3991.75 & 3611.83 & 3515.54 & 3472.87 & 3413.49 & 3816.87 \\
\hline $1998-99$ & 4114.66 & 3911.95 & 3317.49 & 3271.73 & 2988.4 & 3089.88 & 2866.55 & 2912.39 & 2945.99 & 3275.05 & 3289.24 & 3689.42 \\
\hline $1999-00$ & 3455.05 & 3880.37 & 4066.84 & 4526.25 & 4662.84 & 4724.96 & 4835.47 & 4588.53 & 4802.02 & 5407.14 & 5650.66 & 3689.42 \\
\hline $2000-01$ & 4905.3 & 4253.11 & 4675.4 & 4647.34 & 4330.31 & 4416.61 & 3819.69 & 3928.1 & 4081.42 & 4152.39 & 4310.13 & 5261.77 \\
\hline $2001-02$ & 3480.94 & 3613.84 & 3439.01 & 3346.88 & 3304.99 & 2918.28 & 2933.55 & 3164.25 & 3314.88 & 3353.31 & 3528.58 & 3807.64 \\
\hline $2002-03$ & 3435.13 & 3302.91 & 3257.03 & 3214.87 & 3053.16 & 3085.53 & 2949.76 & 3058.19 & 3315.84 & 3327.66 & 3278.85 & 3580.73 \\
\hline $2003-04$ & 3036.66 & 3033.47 & 3386.89 & 3665.46 & 3977.86 & 4314.74 & 4742.32 & 4951.1 & 5424.67 & 5954.15 & 5826.74 & 3155.7 \\
\hline $2004-05$ & 5809.01 & 5204.65 & 4823.87 & 4972.88 & 5144.17 & 5423.27 & 5701.61 & 5960.75 & 6393.83 & 6300.76 & 6595.05 & 6679.18 \\
\hline $2005-06$ & 6379.29 & 6482.72 & 6925.86 & 7336.7 & 7726.03 & 8272.32 & 8220.45 & 8552.09 & 9162.07 & 9539.67 & 10090.08 & 10857.03 \\
\hline
\end{tabular}

Table 1. Monthly average of BSE sensex

\begin{tabular}{|c|c|c|c|c|c|c|c|c|c|c|c|c|}
\hline Year/Month & Apr. & May & Jun. & Jul. & Aug. & Sept. & Oct. & Nov. & Dec. & Jan. & Feb. & Mar. \\
\hline 1 & 2 & 3 & 4 & 5 & 6 & 7 & 8 & 9 & 10 & 11 & 12 & 13 \\
\hline $1990-91$ & 417.99 & 423.79 & 428.68 & 486 & 573.49 & 669.06 & 672.79 & 644.79 & 573.77 & 506.56 & 553.24 & 593.69 \\
\hline $1991-92$ & 627.72 & 641.32 & 639.17 & 703.02 & 825.33 & 872.98 & 853.77 & 898.09 & 880.5 & 960.14 & 1138.17 & 1579.04 \\
\hline $1992-93$ & 1850.94 & 1481.01 & 1351.06 & 1267.88 & 1260.28 & 1444.95 & 1376.05 & 1194.63 & 1162.92 & 1160.02 & 1221.62 & 1081.17 \\
\hline $1993-94$ & 993.63 & 1029.84 & 1057.45 & 1030.1 & 1199.31 & 1283.5 & 1292.72 & 1368.88 & 1589.25 & 1827.17 & 1945.4 & 1842.82 \\
\hline $1994-95$ & 1855.81 & 1822.25 & 1967.76 & 1947.56 & 2080.67 & 2133.49 & 2054.54 & 1968.58 & 1876.13 & 1755.38 & 1683.04 & 1658.97 \\
\hline $1995-96$ & 1631.55 & 1539.44 & 1570.48 & 1550.57 & 1568.33 & 1555.07 & 1603.84 & 1442.44 & 1406.95 & 1369.94 & 1556.09 & 1539.14 \\
\hline $1996-97$ & 1649.6 & 1701.15 & 1771.88 & 1676.6 & 1575.49 & 1522.67 & 1409.83 & 1356.4 & 1290.21 & 1502.66 & 1504.97 & 1629.43 \\
\hline $1997-98$ & 1586.13 & 1610.98 & 1716.56 & 1844.63 & 1863.62 & 1717.52 & 1722.58 & 1563.46 & 1525.78 & 1512.7 & 1467.54 & 1654.92 \\
\hline $1998-99$ & 1804.55 & 1728.93 & 1459.27 & 1437.4 & 1333.8 & 1371.49 & 1281.38 & 1298.19 & 1307.34 & 1452.71 & 1450.6 & 1620.74 \\
\hline $1999-00$ & 1506.84 & 1682.65 & 1755.07 & 1960.83 & 2075.59 & 2156.82 & 2272.13 & 2161.39 & 2429.71 & 2822.05 & 3394.88 & 3109.03 \\
\hline $2000-01$ & 2663.53 & 2120.93 & 2334.27 & 2344.29 & 2180.79 & 2249.43 & 1931.61 & 2017.59 & 2113.84 & 2140.09 & 2203.99 & 1829.32 \\
\hline $2001-02$ & 1641.89 & 1753.46 & 1661.26 & 1572.67 & 1559.95 & 1373.77 & 1357.64 & 1486.33 & 1587.92 & 1601.92 & 1711.43 & 1746.78 \\
\hline $2002-03$ & 1715.11 & 1661.21 & 1658.78 & 1623.07 & 1536.74 & 1521.96 & 1466.79 & 1510 & 1632.19 & 1642.07 & 162.58 & 1559.54 \\
\hline $2003-04$ & 1504.62 & 1538.65 & 1729.15 & 1843.86 & 2055.64 & 2242.97 & 2423.87 & 2543.09 & 2813.58 & 3142.23 & 3003.89 & 2956.07 \\
\hline $2004-05$ & 3101.76 & 2772.81 & 2563.78 & 2653.04 & 2748.23 & 2908.81 & 3049.82 & 3188.92 & 3455.28 & 3403.25 & 3558.11 & 3595.2 \\
\hline $2005-06$ & 3431.03 & 3483.19 & 3697.22 & 3920.12 & 4139.35 & 4407.48 & 4353.07 & 4508.71 & 4825.99 & 5048.54 & 5303.59 & 5686.04 \\
\hline
\end{tabular}

Table 2. Monthly average of NSE index

\section{References}

Bowerman, B. L. \& O’ Connell, R. T. (1987). Time series forecasting. New York PWS.

Box, G. E. P. \& Jenkins, G.M. (1976). Time series analysis, forecasting, and control. San Francisco, CA: Holden-Day.

Cichocki, A. \& Unbehauen, R. (1993). Neural networks for optimization and signal processing. New York: Wiley.

Gallegati, M. (2008). Wavelet analysis of stock returns and aggregate economic activity. Computational Statistics \& Data Analysis, 52(6), 3061-3074.

Kumar, A., Joshi, L. K., Pal, A. K. \& Shukla, A. K. (2010). A new approach for generating parametric orthogonal wavelet. Journal of Wavelet Theory and Applications, 4(1), 1-8.

Kumar, A., Joshi, L. K., Pal, A. K. \& Shukla, A. K. (2011). MODWT based time scale decomposition analysis of BSE and NSE indexes financial time series. International Journal of Mathematical Analysis, 5(27), 1343-1352.

Percival, D. B. \& Walden, A. T. (2000). Wavelet methods for time series analysis. Cambridge University Press.

Razadan, A. (2002). Scaling in the Bombay stock exchange index. Pramana, 58(3), 537-544. 
International Journal of Mathematical, Engineering and Management Sciences

Vol. 1, No. 1, 26-33, 2016

https://dx.doi.org/10.33889/IJMEMS.2016.1.1-003

Saiti, B., Bacha, O., \& Masih, A. (2014). Is the global leadership of the US financial market over other financial markets shaken by 2007-2009 financial crisis? Evidence from Wavelet Analysis. University Library of Munich, Germany.

Schleer-van Gellecom, F. (2014). Advances in non-linear economic modeling-theory and applications. In Dynamic Modeling and Econometrics in Economics and Finance. (Vol.17), Springer.

Weigend, A. S. \& Gershenfeld, N.A. (1994). Time series prediction: Forecasting the future and understanding the past. Reading, MA: Addison-Wesley. 